stopped, and the whole body became cyanotic. Fach fit lasted for about 10 seconds; then the face lost its grin, the child became of a better colour, the sterno-mastoids relaxed somewhat, and the arms could be moved from the shoulder and slightly from the elbow. The legs did not get so relaxed but the orthotonos disappeared.

The seat of the trouble was a septic and pervious umbilicus. But in addition there was severe ophthalmia neonatorum. The umbilicus was curetted and dressed antiseptically under chloroform and the ophthalmia was treated with silver nitrate (two grains to one ounce). An attempt, however, to grow the tetanus bacillus from the umbilical pus was not successful. Three cubic centimetres of antitotanic serum were injected every four hours and the child was fed by the rectum during the first day, as every attempt to pass the nasal tube brought on a very severe fit. Two grains of bromide of potassium and one minim of liquor morphinæ hydrochloridi were added to each four-hourly feed.

On July 30th the temperature had been rising steadily since admission, reaching $1032^{\prime}$ at $2 \mathrm{~A} . \mathrm{M}$. on the morning of the 31st. Fits occurred every five minutes. The patient was fed by the nasal tube under chloroform. 18 cubic centimetres of anti-tetanic serum were injected during the day (three cubic centimetres every four hours). On the 31st a pink rash due to the antitoxin came out all over the body. The child seemed better, however, as the respirations were not so hurried, the fits were less frequent, and the rigidity between the attacks was not so well marked. The temperature, however had been going up, reaching $106^{\circ}$ at $2 \mathrm{~A}$.M. on the morning of August 1st the highest point reached during the acute stage. He was fed by the nasal tube without anæsthetic, though each time an attack was brought on. 18 cubic centimetres of serum were injected during the day. On August 1st the temperature had fallen to $99^{\circ}$ during the night. The fingars could be moved slightly and tri-mus was not so constant. On the 2 nd the patient was fed by the stomach-tube. On the 3rd the temperature was subnormal for the first time. The serum was discontinued. The breathing was much easier. He still had a fit each time he was fed, but he had no other fits between. There were no fits on the 5th. The abdomen and limbs were still rigid. On the 6th he took one ounce of milk extra by the spoon. On the 11th he was fed by the bottle and on the 28 th he was discharged recovered.

Remarks by Dr. ABRAHAM. - The comparative rarity of tetanus neonatorum, its almost constant fatal termination, and the usually unsatisfactory results obtained from injection of anti-tetanic serum in acnte cases, justify me in recording the above case in which recovery took place, and the serum appeared to have done some good. The disease would seem to be fairly common in tropical countries. especially in the West Indies, but it is comparatively rare in European countries. It is stated, however, that one out of every six children born in the Rotunda Hospital, Dublin, in 1782 died from the disease. In more recent times it was exceedingly fatal in St. Kilda in the Western Hebrides, where it was known as the "eight days" sickness"-84 out of 125 children dying within 14 days (Osler); but with this exception it is now very rare, as, according to Ware, "a case has not been admitted to St. Bartholomew's during the period covered by the statistical reports." 1 As to the fatality, no exact stati-tics are obtainable, the infantile form being included in the general statistics of tetanus. These show that for an incubation period of 10 days 4 per cent. of the patients recover, and for an incubation period of from 11 to 15 days 27 per cent. recover (Rose). With regard to the serum treatment, the general consen-us of opinion, voiced by Kanthack, Roux, Nocard, and others, is summed up by Rose thus: "In man it has not been conclusively proved that recovery has ever been due to serum. Local antiseptics, narcotics, and strengthening treatment hold out the best hopes of recovery."2

\section{EASTERN FEVER HOSPITAL.}

A CASE OF PURPURA FOLLOWING DIPHTHERIA. (Under the care of Dr. E. W. GoodalL and Dr. ConRad BASAN.)

THE rarity of post-diphtheritic purpura is very great. We are so little acquainted with the esrential cause of any form of purpura that it is of little value to speculate on the method of production of the purpura in this case. It is probable, however, that in all cases there is a modification nut only in the coagulability of the blood, but also in the walls of the capillaries.

A girl, aged 11 years, was admitted to the Eastern Fever Hosuital on Sept. 16th, 1901, for diphtheria, of which the symptoms had appeared on the previons day. On the day of admission there was a thick layer of membrane on each tonsil, the cervical glands were enlarged, and the temperature was $1036^{\circ} \mathrm{F}$. Antitoxin (6000 units) was injected and the fauces were flushed with an alkaline solution every four hours. The progress of the disease was favourable and on the 22nd the membrane had quite disappeared. On the 23rd and 24th there was urticaria of the trunk, face, and limbs, without any rise of temperature, but on the 25 th this ras $h$ had disappeared. On the 26 th there was again a slight erythematous rash but no pyrexia. On the 27 th a purpurie eruption made its appearance upon both the upper and lower extremities. chiefly about the elbows and the front of the legs. There were a few spots on the trunk and there was some hæmorrhage beneath the right conjunctiva. Mixed up with the spots on the skin was a circinate erythema. There was also bleeding from the nose and mouth, and pin-point hæmorrhages were observed in the buccal mucous membrane. The temperature was $100: 2^{\circ}$ and the pulse-rate was 130 . On the 28th fresh purpuric spots appeared on the face as well as on the trunk and extremities. After this no further spots came out. Most of the spots were cutaneous, small in size, and of a purple hue, but a few were subcutaneous, larger, and of a blue colour. A few days prior to the attack of diphtheria the patient had been vaccinated on the left arm and at the time the purpura appeared the pocks were in the vesicular stage. Hæmorrhage now took place into and around the pocks, which after a day or two looked as if they would slough, but no such event occurred and the scabs reparated in the usual way on Oct. 7th. In addition to bleeding from the mouth and nose bright blood was $n n$ two or three occasions passed per anum on Sept. 27th and 28th. On the 29th the hæmorrhages were already beginning to fade and the larger ones were of a greenish-brown hue, like old bruises. On this day the circinate erythema was still present. During the day the patient complained of pain in some of the joints and her temperature rose to $1026^{\circ} \mathrm{F}$. The pains in the joints were worse the next day, the large joints being chiefly affected. There was, indeed, some effusion into the right knee-joint. By Oct. 3rd this joint affection had quite gone. On Sept. 27th and 28th, while the hæmorrhages were coming out, the girl was very restless and anxious, complained much of thirst, and slept little. There were also nausea and occasional vomiting. On Oot. 1st the temperature became normal. After the 3 rd recovery was fairly rapid, though from the 5 th to the 15 th there were pain and tenderness along the course and distribution of the ulnar nerve. There was never albuminuria at any time during the patient's stay in the hospital. She was discharyed well on Oct. 24th. As for the treatment, calcium chloride, five grains every four hours, was given on the appearance of the jurpura and was continued till Sept. 30th. Pain was relieved by opium and phenalgine. The nausea and vomiting necessitated the administration of food by means of nutrient enemata for two or three days.

Remarks by Dr. GoodaLL. - I bave ventured to record this case for two reasons. Fir.tly, because of its rarity ; I have seen only three such cases, including the present one. The account of the first will be found in Vol. L. of the "Guy's Hospital Reports" ; and of the other in Appendix I. to the "Report on the Antitoxin Treatment" made by a committee of the Clinical Society of London in 1898. A similar but more severe case was published in THE LANCET of July 20th, 1901 , p. 132, by Dr. C. W. Buckley. Secondly, these rare ca:es of purpura following diphtheria must be distinguished from those common cases in which hæmorrhages, both into the skin and from mucous membranes, occur during the acute stage of the disease (hæmorrhagic diphtheria). Practically every case of hæmorrhagic diphtheria is fatal, whereas in cases of post-diphtherial purpura the prognosis is by no means always grave. In fact, my three cases all recovered, as also did that reported by Dr. Buckley. Dr. Buckley raised the question whether antitoxin has any share in the production of the purpuric symptoms. I do not think it has. My first case occurred befure the antitoxin treatment was introduced, and I have met with a similar purpura following scarlet fever where also no antitoxin had been given.

For the notes of the case I am indebted to Dr. Basan, 
assistant mediaal officer, under whose immediate care the potient was.

\section{BROMLEY COTTAGE HOSPITAL, KENT.}

1 CASI IN WHICH THE VULCANITE MOUTHPIKCE OF A PIPE WAS IMPACTED IN THE CHEEK AND PENRTBATED THE FLOOR OF THE ORBIT.

(Under the care of Dr. Herbert J. IlotT.)

Tuz orbit is singularly tolerant of foreign bodies, and sereral instances are recorded of the impaction of tobaccopipes in that cavity.' An important point that should be borne in mind is the tendency these foreign bodies have to pierce the orbital roof and so to cause serious or even fatal injury to the brain. ${ }^{2}$

$\triangle$ man, aged 42 years, was cycling downhill on March 18th while smoking a briar-root pipe with a vulcanite mouthpiece. The chain of the machine became detached and he was pitched forward over the handle-bar, falling into the road. He fell on his left side, sustaining a Colles's fracture of the left wrist and felt pain in the left side of his face. He noticed that the pipe was broken and threw away the bowl and wooden part of the stem, thinking that the mouthpiece was in the road, but no search was made for it. On the same evening he went to his club surgeon who attended to his wrist, applying splints. His mouth was bleeding from a wound on the inner side of the right cheek on the level of the first molar tooth, but no close examination was made of the injury inside the mouth. The face became very swollen and painful, the eyelids being so swollen that for several days he could not see out of the right eye. When the swelling of the lids had somewhat subsided he noticed that the eyeball was prominent and he had double vision. It was thought that the bone was fractured and that an abscess was forming, but no suspicion of the presence of a foreign body seems to have been entertained by the patient or his medical man. He was then sent to the Westminster Ophthalmic Hospital. He was told that the cheek wanted attention but that when that was righted the eye trouble would disappear. This was on April 3rd; the wound in the mouth was discharging pus at the time. He returned home and continued the use of poultices and of fomentations which he had been ordered. At times be suffered much pain.

On April 21,t he was sent to Dr. Ilott by his medical man. Dr. Ilott noticed the protrusion of the eyeball and felt a hardness in the right cheek. On the inside of the mouth was a sinus discharging pus. On introducing a probe a hard smooth subitance was felt and on moving the probe to one side it sank into what felt like a fissure in the bone. On the 24th Dr. Ilott again examined him at the Bromley Cottage Hospital. On again probing the instrument struck against the hard substance and passed in some distance. On withdrawing it the end was noticed to be discoloured and smelt of nicotine. An incision was then made through the mucous membrane for about halt an inch and on being deepened and inspected a circular black surface was exposed. Introducing a pair of necrosis forceps this was seized and a black vulcanite tobacco-pipe mouthpiece measuring two and seven-eighth inches was withdrawn. A probe passed along the track went through an opening in the bone into the orbit. The cavity was syringed out with weak perchloride solution and drained.

On May 3rd the patient was shown at a meeting of the North Kent District of the South-Eastern Branch of the British Medical Association at Dartford, and his case evoked much interest from the members assembled. On the 8th he was again seen at the Westminster Ophthalmic Hospital and the proptosis had nearly disappeared and vision was much improved. On the 14 th Dr. llott saw him again. The tract bad healed, the induration in the substance of the cheek had also gone. and the sight was normal.

Remarks by Dr. ILOTT. - The case is of interest from the fact of a foreign borly of so large a size being impacted unsuspected for so long a time-nearly five weeks-and the rapid recovery and relief that followed its removal. 1. Tre Laxokr, July 4th, 1891, p. 15. Brit. Med. Jour., 1880, vol. i.,
p. 514 . 2 Tre Lasaer, July 17th, 1886, p. 143.

\section{Sttedital Socitetirs.}

\section{ROYAL MEDICAL AND CHIRURGICAL SOCIETY.} Intestinal Obstruction due to the Pressure of a Vesioal
Sacculus upon a Coil of \$mall Intestime.-Allaptonuria.

A MEeting of this society was held on Nor. 26th, Dr. C. TheOdore Williams, the Vice-President, being in the chair.

Mr. THOMAs BRYaNT described a case of Intestinal Obstruction due to the Pressure of a Vesical Sacculus upon a Coil of Small Intestine in a man, aged 67 years, who during a brief illness from angina pectoris became the subject of intestinal obstruction, associated with a tumour rising from the pelvis and extending upwards in the abdomen to the right of the median line above the level of the umbilicus. Symptoms had gradually increased in severity for eight days, obstruction had become complete, and the vomit had become fæcal. An exploratory incision was made and it was found that the obstruction was due to the presence of a vesical sacculus, springing from the right side of the fundus of the bladder; this had by pressure upon a coil of small intestine caused complete obstruction. The existence of an abduminal tumour had been recognised by Dr. M. G. Biggs, the patient's medical attendant, at least one and a half years previously when he had been called in for some passing bladder trouble. It was then but small, only just rising out of the pelvis; at that time some enlargement of the prostate gland was also recognised. The patient survived the operation only 22 hours. No necropsy was permitted. The case was an unusual one, for it seemed certain that the cause of the patient's intertinal obstruction had been due to the pressure of the sau-age-shaped vesical sacculus upon a coil of small intestine which passed behind it and between it and the spinal column, and this view was supported by the fact that on opening the abdomen the colon and small intestire on the right of the tumour were found pale and empty, whereas the small intestine on the left side was found full and congested, the seat of pressure upon the bowel by the tumour being very evident. It was likewi-e clear that the opening of communication between the sacculus and the bladder must have been very minute, for the bladder seemed to have done its duty during the formation of the sacculus in apparently a satisfactory way, and even at the time of operation, when the bladder was emptied by means of a catheter, the tension in the sacculus was not materially affected, for it was only upon manual compression of the sacculus that it was emptied, and then but slowly. It should not, therefore, be forgotten that a vesical sacculus might be the cause of intestinal obstruction.Mr. Reginald Harrison said that he knew of two cases which were of interest in connexion with that reported by Mr. Bryant. In the first case, which was one of vesical sacculus, there had been during the course of the illness remarkable and unexplained attacks of con-tipation. An operation was performed and it was found that the sacculus came in contact with, and pressed upon, the rectum, and it was clear that the constipation was due to this cau-e, for subsequently the patient ceased to be subject to these attacks. The second case was also one in which a vesical sacculus was diagnosed. The patient was in a very feeble condition. The sacculus was drained, but the patient died, and it was found that the apex of the sacculus was adherent to the intestine and that the lumen of the gut at this point was reduced to about one-half its normal diameter.Mr. Clinton T. DENT said that it would be of interest to know if there was any stenosis of the intestine in Mr. Bryant's case. He thought that it was most unusual for a sacculus of the bladder in which there was no inflammation to cause obstruction of the intestine.Mr. BRYANT, in reply, said that the first case mentioned by Mr. Harrison was very similar to the one which he had recorded, but the second case differed in that the sacculus was adherent to, and had caused actual narrowing of, the intestine. With regard to the condition of the inte-tine in his case it was clear that there was no actual stricture, for as swon as the bladder tumour was raised the contents of the distended intestine passed into the empty intestine. Tho 